$\begin{array}{ll}\text { Lung } & \text { respiration } \\ \text { Mikity-Wilson } & \text { respiratory distress } \\ \quad \text { syndrome } & \text { syndrome } \\ \text { premature infant } & \end{array}$

\title{
Ventilation-Perfusion Abnormalities in the Premature Infant: Triple Gradient
}

\author{
A.N. Krauss and P.A.M. Auld ${ }^{[12]}$ \\ Department of Pediatrics, Cornell University Medical College, New York, N.Y., USA
}

\begin{abstract}
Extract
Serial studies of arterial and alveolar oxygen, carbon dioxide, and nitrogen tensions, arterial $\mathrm{pH}$, and ratios of dead space: tidal volume were made in 24 nondistressed premature infants. By determining arterial-alveolar gradients, the sequence of development of ventilation/perfusion relations in the nondistressed premature infant can be determined. The presence of an arterial-alveolar carbon dioxide gradient and elevated $\mathrm{V}_{\mathrm{D}} / \mathrm{V}_{\mathrm{T}}$ ratio indicate the presence of overventilated/underperfused areas. Alveolar-arterial oxygen gradients may be due to shunting, underventilation (low ventilation/perfusion ratio), or diffusion block. Although underventilation exists, as evidenced by the presence of a urinary-alveolar gradient for $\mathrm{N}_{2}$, it is not a major cause of arterial unsaturation. This evidence suggests that shunting of blood, either through intracardiac shunts or atelectatic lung, is a major cause of arterial unsaturation in the premature infant.
\end{abstract}

\section{Speculation}

Further extension of this work should make possible the construction of $\dot{\mathrm{V}}_{\mathrm{A}} / \dot{\mathrm{Q}}$ curves for the individual patient and thus show in even greater detail the maturation of pulmonary ventilation and perfusion.

\section{Introduction}

The onset of respiration at birth requires adjustments in ventilation and perfusion of lungs. Inequalities in the ventilation-perfusion ratio $\left(\dot{\mathrm{V}}_{\mathrm{A}} / \dot{\mathrm{Q}}\right)$ will result in gradients between alveolar and arterial oxygen tension $\left(\mathrm{AaD}_{\mathrm{O}_{2}}\right)$, arterial-alveolar carbon dioxide tension $\left(\mathrm{aAD}_{\mathrm{CO}_{2}}\right)$ and arterial-alveolar nitrogen tension $\left(\mathrm{aAD}_{\mathrm{N}_{2}}\right)$. Assessment of these parameters, the so-called triple gradient, permits description of the nature of the abnormalities of ventilation and perfusion. This study investigated these gradients serially in a group of small nondistressed premature infants in order to determine the sequence of events in the adaptation of lungs to extrauterine life. Serial measurements of anatomic and physiologic dead space and calculation of alveolar dead space were made, since these also are indications of the adequacy of ventilation-perfusion.

\section{Materials and Methods}

The subjects were twenty-four premature infants, all but one of whom had a birth weight less than $1800 \mathrm{~g}$. With one exception, the infants were of appropriate weight for gestational age. Four infants recovering from hyaline membrane disease and two with roentgenographic findings of the Mikity-Wilson syndrome 
were the only ones with symptoms of respiratory difficulty, although some of the smaller infants required an increased oxygen environment for several days after birth.

Alveolar-arterial oxygen gradients $\left(\mathrm{AaD}_{\mathrm{O}_{2}}\right)$ and arterial-alveolar carbon dioxide gradients $\left(\mathrm{aAD}_{\mathrm{CO}_{2}}\right)$ were determined by methods previously reported [7]. The nitrogen gradient was measured as the urinaryalveolar nitrogen gradient $\left(\mathrm{uAD}_{\mathrm{N}_{2}}\right)$ by the method of FARHI $e t$ al. [1]. Urine was collected in a polyethylene bag attached to the perineum of the infant. On voiding, the bag was immediately removed and a clamp was placed below the air-liquid interface. Urine was then anerobically removed from the bag into a $5-\mathrm{ml}$ syringe. Dead space of the syringe had been previously filled with distilled water and rinsed once with urine. Rectal temperature was recorded immediately with a telethermometer [10]. Pu $\mathrm{N}_{2}$ was determined from measurement of dissolved nitrogen in an anerobically collected specimen and the nitrogen content dissolved in an aliquot of the same sample which had been equilibrated with a gas of known $\mathrm{P}_{\mathrm{N}_{2}}$ at the same temperature as that of the infant. The nitrogen content of the samples was determined using a Beckman GC-2A gas chromatograph [1]. A volume of $0.07 \mathrm{ml}$ of urine was used for each determination and approximately $4 \mathrm{ml}$ was needed for both anerobic and equilibrated samples. A peak height of approximately $15 \mathrm{~cm}$ was obtained for each determination. Triplicate checks within $2.5 \mathrm{~mm}$ peak height were required and the mean value was used to calculate $\mathrm{Pu}_{\mathrm{N}_{2}}$. The equilibrated sample was obtained by using a Twin tonometer for tonometering the urine with compressed air of known nitrogen content for 30 minutes at the exact rectal temperature of the infant. Urinary nitrogen was calculated from the mean peak height of the anerobic sample, the mean peak height of the equilibrated sample, and the partial pressure of nitrogen in the equilibrated urine as follows:

$$
\mathrm{Pu}_{\mathrm{N}_{2}}=\frac{\text { peak height (anerobic) }}{\text { peak height (equilibrated) }} \times \underset{\text { (equilibrated) }}{\mathrm{P}_{\mathrm{N}_{2}}}
$$

The partial pressure of nitrogen in alveolar gas $\left(\mathrm{PA}_{\mathrm{N}_{2}}\right)$ was determined from the alveolar air equation using the measured $\mathrm{PA}_{\mathrm{CO}_{2}}$ and $\mathrm{R}$ equal to 0.7 in the first week of life, and $R$ equal to 0.8 thereafter [2]. The uAD $\mathrm{N}_{2}$ was obtained by using the equation: $\mathrm{uAD}_{\mathrm{N}_{2}}=$ $\mathrm{Pu}_{\mathrm{N}_{2}}-\mathrm{PA}_{\mathrm{N}_{2}}$.

In some infants, physiologic dead space $\left(V_{D}\right)$, tidal volume $\left(V_{T}\right)$, dead space tidal volume ratio $\left(V_{\mathrm{D}} / \mathrm{V}_{\mathrm{T}}\right)$, and alveolar ventilation $\left(\dot{V}_{\mathrm{A}}\right)$ were measured. For these studies, carbon dioxide production $\left(\dot{\mathrm{V}}_{\mathrm{CO}_{2}}\right)$ was determined by using a Kipp and Zonen diaferometer, with the infant's environmental temperature at the neutral range. Tidal volume was measured by electron- ically integrating the signal obtained from a pneumotachograph, the dead space of which was obliterated by a constant flow through of air [4]. Volumes were obtained from standard respiratory equations. The respiratory rate measured at the time of determination of $\dot{\mathrm{V}}_{\mathrm{CO}_{2}}$ was used for the calculations.

\section{Evaluation of Nitrogen Gradient Data}

The validity of the method of determination of the nitrogen gradient has been discussed previously [3]. In the present study, the smaller amount of urine used may have decreased the accuracy of the test. Thirteen determinations on a normal adult, however, showed a mean value of $4 \mathrm{~mm} \mathrm{Hg}$ for $u A D_{N_{2}}$ with a range -9.3 to 12.3, and one determination on an adult with emphysema showed a value of $22 \mathrm{~mm} \mathrm{Hg}$. The assumption that body temperature at the time of urine formation was the same as at the time of collection may not be valid. Many of the infants were in isolettes, and there was some variation in body temperature. The relation $\dot{\mathrm{V}}_{\mathrm{A}} / \mathrm{Q}$ may possibly have changed between the time of urine formation and urine collection. The frequent sighs of premature infants may result in a constantly changing $\dot{\mathrm{V}}_{\mathrm{A}} / \dot{\mathrm{Q}}$. These factors may explain some of the variations noted. There were several negative gradients that could not be explained, although such values have been noted by others.

\section{Results}

Data relating to serial studies appear in tables I and II and in figures 1 and 2. Initial values for $\mathrm{AaD}_{\mathrm{O}_{2}}$ ranged from 25 to $60 \mathrm{~mm} \mathrm{Hg}$ in nondistressed infants. The gradients decreased with age so that most infants had a value of 20 to $30 \mathrm{~mm} \mathrm{Hg}$ by 60 days of age. Exceptions included two infants (Nos. 1 and 13) who developed the Mikity-Wilson syndrome (table I). These infants had values as high as $50 \mathrm{~mm} \mathrm{Hg}$ as late as 104 days of age. Except for one infant (No.24) who had respiratory distress (table $\mathrm{I}$ ), the value for $\mathrm{aAD}_{\mathrm{CO}_{2}}$ on the first day of life was $0-12 \mathrm{~mm} \mathrm{Hg}$. This gradient tended to rise during the first week of life and then gradually fell, except in infants Nos. 1 and 13. Wide daily variations were found in values for $\mathrm{uAD}_{\mathrm{N}_{2}}$, but a general trend toward a low value with time was noted. Most infants were found initially to have a high ratio of $V_{\mathrm{D}} / \mathrm{V}_{\mathrm{T}}$ (fig. 3). There was a concomitant fall in the ratio of $\mathrm{V}_{\mathrm{D}} / \mathrm{V}_{\mathrm{T}}$ and in $\mathrm{aAD}_{\mathrm{CO}_{2}}$. In most infants, this decrease was related to a rise in effective alveolar ventilation and a fall in alveolar dead space. Anatomic dead space generally increased as the infant grew in size. Some infants had over 25 percent nonperfused 
Table I. Serial measurements of gradients for $\mathrm{O}_{2}, \mathrm{CO}_{2}$, and nitrogen ${ }^{1}$

\begin{tabular}{|c|c|c|c|c|c|c|c|c|c|c|c|c|c|c|c|}
\hline $\begin{array}{l}\text { Case } \\
\text { No. }\end{array}$ & $\begin{array}{l}\text { Age } \\
\text { days }\end{array}$ & $\begin{array}{l}\text { Birth } \\
\text { weight } \\
\mathrm{g}\end{array}$ & $\begin{array}{c}\text { Weight }{ }^{2} \\
\mathrm{~g}\end{array}$ & $\begin{array}{l}\text { Length } \\
\text { cm }\end{array}$ & $\mathrm{PA}_{\mathrm{O}_{2}}$ & $\mathrm{~Pa}_{2}$ & $\mathrm{AaD}_{\mathrm{O}_{2}}$ & $\frac{\mathrm{PaCO}_{2}}{\mathrm{~mm} \mathrm{Hg}}$ & $\mathrm{PA}_{\mathrm{CO}_{2}}$ & $\mathrm{aAD} \mathrm{CO}_{2}$ & $\mathrm{uAD} \mathrm{N}_{\mathrm{N}_{2}}$ & $\mathrm{pH}$ & $\mathrm{Hct}$ & $\begin{array}{l}\text { Body } \\
\text { temp. } \\
{ }^{\circ} \mathrm{C}\end{array}$ & Remarks \\
\hline \multirow{8}{*}{1} & 34 & & 1030 & 35 & 106.4 & 45.0 & 61.4 & 56.5 & 35.3 & 21.2 & 17.9 & 7.353 & 20 & 37.3 & Mikity-Wilson \\
\hline & 35 & & 1020 & & & & & & & & 32.1 & & & & \\
\hline & 39 & & & & & & & & & & 12.6 & & & & \\
\hline & 46 & & 1210 & 36 & & 40.7 & & 55.5 & & & 33.6 & 7.370 & 25 & 37.3 & \\
\hline & 61 & & 1540 & 40 & 105.5 & 49.9 & 55.6 & 56.4 & 35.4 & 21.0 & 10.5 & 7.453 & 26 & 36.8 & \\
\hline & 69 & & & & & & & & & & 6.8 & & & & \\
\hline & 76 & & 1830 & 40 & 107.8 & 48.1 & 59.7 & 43.6 & 34.8 & 8.8 & 5.7 & 7.403 & 23 & 36.8 & \\
\hline & 104 & & 2560 & 46 & 111.6 & 42.9 & 68.7 & 42.1 & 29.5 & 12.6 & 2.5 & 7.445 & 23 & 36.8 & \\
\hline \multirow[t]{2}{*}{2} & 1 & 860 & 930 & & 123.4 & 61.0 & 62.4 & 28.8 & 21.5 & 7.3 & 10.0 & 7.470 & 38 & 36.1 & small for gestational \\
\hline & 6 & & 925 & 37 & 126.6 & 59.5 & 67.1 & 31.8 & 20.3 & 11.5 & 1.8 & 7.456 & 43 & 36.2 & age \\
\hline \multirow[t]{4}{*}{3} & 2 & 1100 & 1130 & & 104.6 & 61.9 & 42.7 & 44.2 & 38.4 & 5.8 & 4.3 & 7.354 & 30 & 36.5 & died—8th day \\
\hline & 3 & & 1070 & & 99.1 & 67.2 & 31.9 & 39.3 & 43.3 & & & 7.355 & 25 & 35.9 & sepsis and \\
\hline & 4 & & 1070 & & & & & & & & & & & & enterocolitis \\
\hline & 6 & & & & & & & & & & -3.8 & & & & \\
\hline \multirow[t]{10}{*}{4} & 3 & 1130 & 1050 & & & & & & & & 9.7 & & & & \\
\hline & 9 & & 990 & & & & & & & & 14.7 & & & & \\
\hline & 10 & & 990 & 38 & 106.9 & 52.0 & 54.9 & 40.0 & 36.4 & 3.6 & & 7.315 & 26 & 36.4 & \\
\hline & 24 & & 1100 & 38 & 108.1 & 67.6 & 30.2 & 37.8 & 34.9 & 2.9 & 10.8 & 7.405 & 23 & 36.8 & \\
\hline & 31 & & & & & & & & & & 4.7 & & & & \\
\hline & 44 & & 1420 & 41 & 118.2 & 89.4 & 26.8 & 34.2 & 28.1 & 5.1 & 5.8 & 7.315 & 24 & 37.1 & \\
\hline & 52 & & 1590 & 42 & 111.4 & 71.6 & 39.8 & 35.0 & 32.2 & 2.8 & 22.1 & 7.487 & 25 & 36.0 & \\
\hline & 53 & & 1600 & & 111.9 & 66.7 & 45.2 & 38.4 & 35.0 & 3.4 & & 7.410 & & & \\
\hline & 54 & & & & & & & & & & 3.1 & & & & \multirow{2}{*}{$\begin{array}{l}{ }^{2} \text { Weight of time } \\
\text { of study }\end{array}$} \\
\hline & 67 & & 2120 & 44 & 116.7 & 84.5 & 32.3 & 33.0 & 29.4 & 3.6 & -2.0 & 7.443 & 22 & 36.2 & \\
\hline
\end{tabular}


Table I. (Continued)

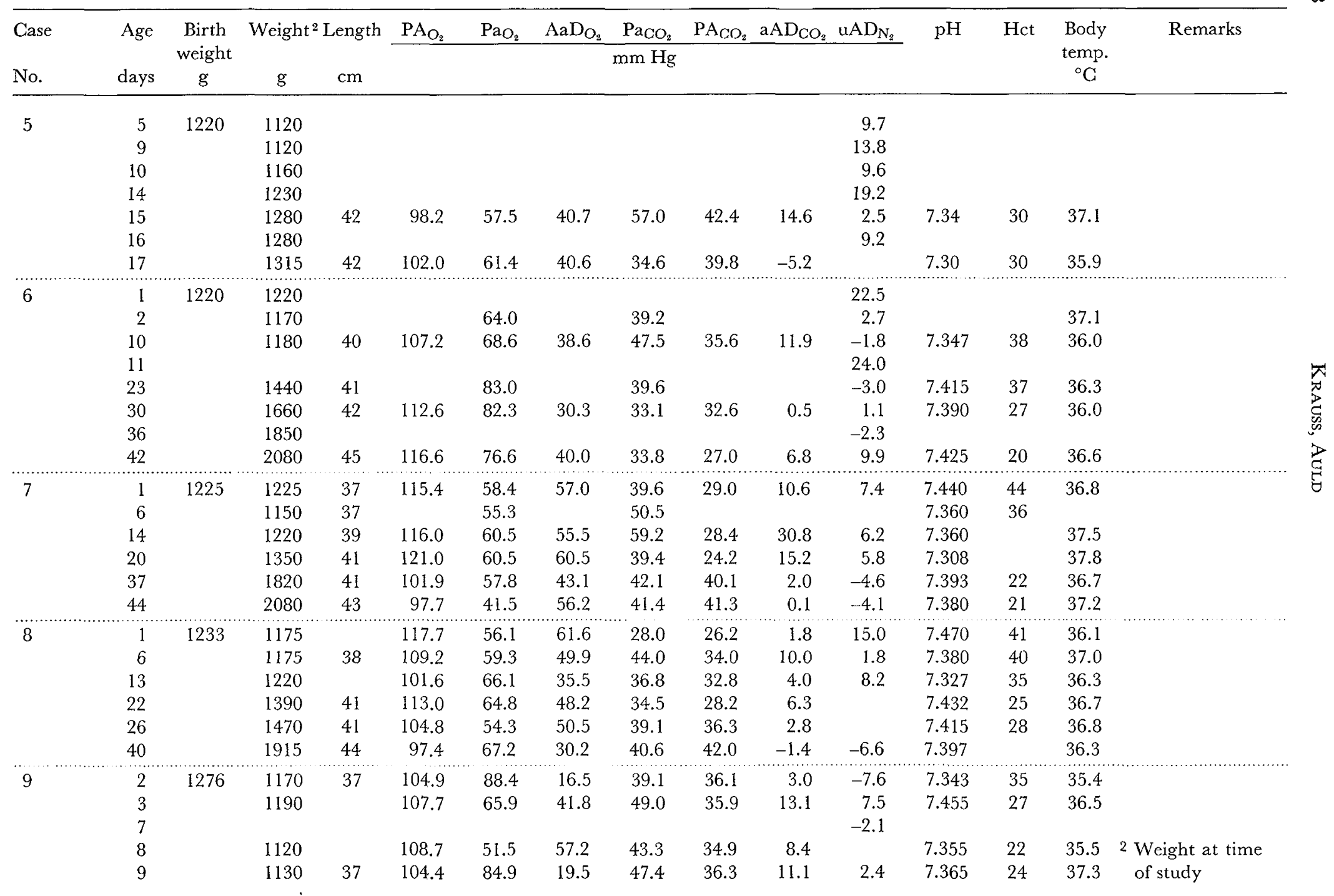




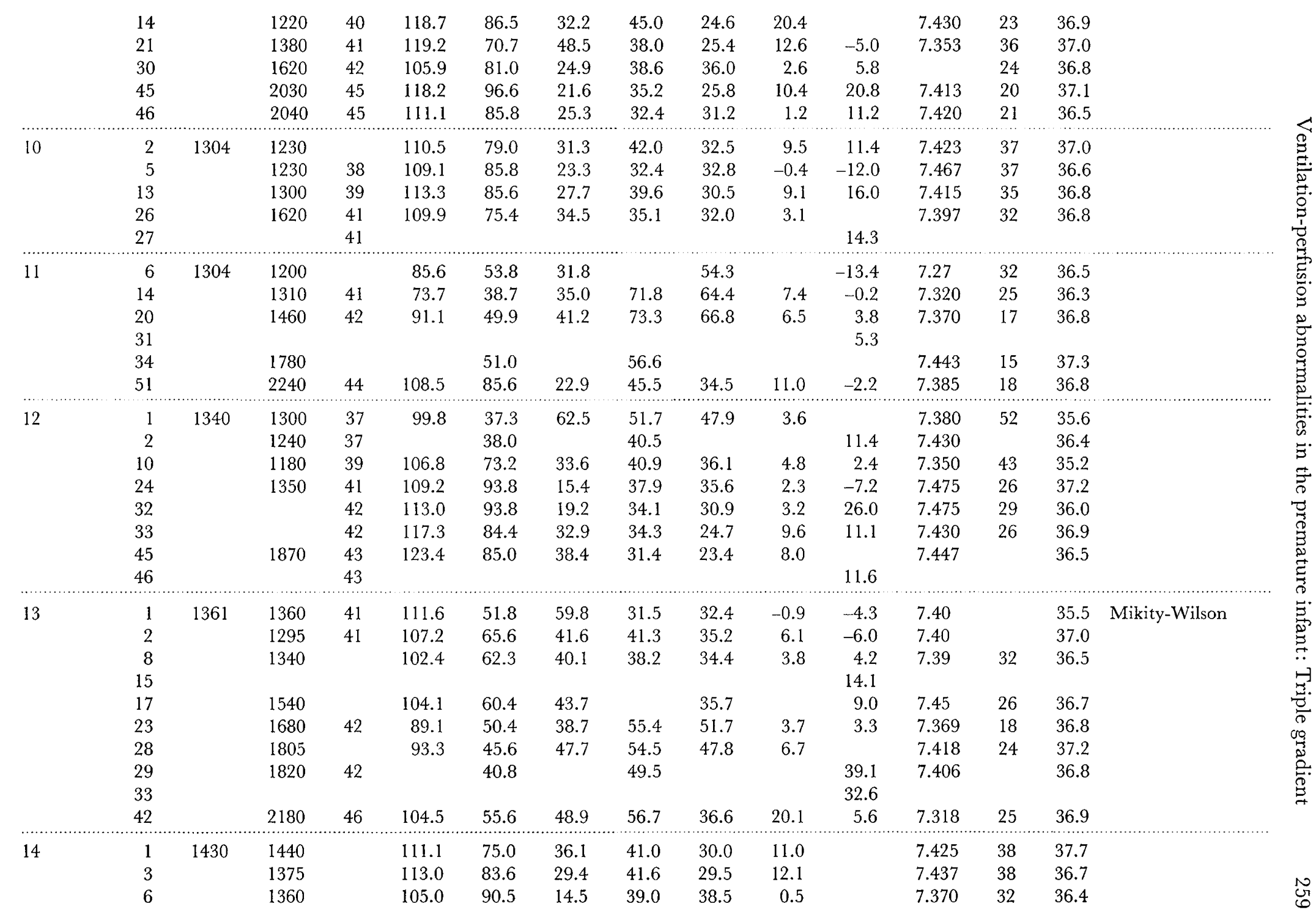


Table I. (Continued)

\begin{tabular}{|c|c|c|c|c|c|c|c|c|c|c|c|c|c|c|c|}
\hline $\begin{array}{l}\text { Case } \\
\text { No. }\end{array}$ & $\begin{array}{l}\text { Age } \\
\text { days }\end{array}$ & $\begin{array}{c}\text { Birth } \\
\text { weight } \\
\text { g }\end{array}$ & $\begin{array}{c}\text { Weight }^{2} \\
\text { g }\end{array}$ & $\begin{array}{l}\text { Length } \\
\qquad \mathrm{cm}\end{array}$ & $\mathrm{PA}_{\mathrm{O}_{2}}$ & $\mathrm{~Pa}_{2}$ & $\frac{\mathrm{AaD}_{\mathrm{O}_{2}}}{\mathrm{n}}$ & $\frac{\mathrm{Pa}_{\mathrm{CO}_{2}}}{\mathrm{~nm} \mathrm{Hg}}$ & $\mathrm{PA}_{\mathrm{CO}_{2}}$ & $\mathrm{aAD}_{\mathrm{CO}_{2}}$ & $\mathrm{uAD}_{\mathrm{N}_{2}}$ & $\mathrm{pH}$ & Hct & $\begin{array}{l}\text { Body } \\
\text { temp. } \\
{ }^{\circ} \mathrm{C}\end{array}$ & Remarks \\
\hline & $\begin{array}{l}11 \\
26\end{array}$ & & 1480 & $\begin{array}{l}40 \\
40\end{array}$ & 113.8 & 86.4 & 27.4 & 34.3 & 29.7 & 4.6 & $\begin{array}{l}24.6 \\
10.2\end{array}$ & 7.300 & 32 & 37.3 & \\
\hline & 28 & & 1820 & 42 & 105.0 & 103.0 & 2.0 & 33.5 & 37.5 & 4.0 & & 7.405 & 30 & 36.4 & \\
\hline 15 & $\begin{array}{r}5 \\
13 \\
19 \\
20 \\
21\end{array}$ & 1474 & $\begin{array}{l}1380 \\
1460 \\
1680 \\
1710\end{array}$ & $\begin{array}{l}40 \\
40 \\
41 \\
41\end{array}$ & $\begin{array}{l}105.5 \\
111.1 \\
112.4\end{array}$ & $\begin{array}{r}65.2 \\
91.5 \\
97.9 \\
104.2\end{array}$ & $\begin{array}{r}16.5 \\
13.2 \\
8.2\end{array}$ & $\begin{array}{l}41.7 \\
44.5 \\
34.2 \\
33.0\end{array}$ & $\begin{array}{l}34.6 \\
33.7 \\
32.2\end{array}$ & $\begin{array}{l}9.9 \\
0.5 \\
0.8\end{array}$ & $\begin{array}{r}16.1 \\
-8.0 \\
-2.5 \\
6.2\end{array}$ & $\begin{array}{l}7.368 \\
7.423 \\
7.433 \\
7.530\end{array}$ & $\begin{array}{l}33 \\
32 \\
30\end{array}$ & $\begin{array}{l}37.1 \\
37.0 \\
36.5 \\
35.9\end{array}$ & \\
\hline 16 & $\begin{array}{r}1 \\
2 \\
16 \\
17 \\
32 \\
34 \\
34 \\
38 \\
41\end{array}$ & 1474 & $\begin{array}{l}1390 \\
1390 \\
1450 \\
1810 \\
1950 \\
1950 \\
2200\end{array}$ & 43 & $\begin{array}{r}108.6 \\
104.3 \\
97.4\end{array}$ & $\begin{array}{l}85.2 \\
76.1 \\
69.7 \\
88.8 \\
89.0 \\
83.0\end{array}$ & $\begin{array}{l}23.4 \\
28.1 \\
27.7\end{array}$ & $\begin{array}{l}40.0 \\
39.9 \\
43.2 \\
\\
38.4 \\
48.0 \\
42.0\end{array}$ & $\begin{array}{l}34.7 \\
38.4 \\
43.2\end{array}$ & $\begin{array}{l}5.3 \\
1.5 \\
0.0\end{array}$ & $\begin{array}{r}-9.9 \\
-7.8 \\
14.0 \\
7.4 \\
8.2 \\
\\
22.5 \\
1.6\end{array}$ & $\begin{array}{l}7.39 \\
7.37 \\
7.380 \\
7.370 \\
7.385 \\
7.358\end{array}$ & $\begin{array}{l}38 \\
37 \\
20 \\
\\
23 \\
20 \\
20\end{array}$ & $\begin{array}{l}35.3 \\
35.7 \\
36.1 \\
36.6 \\
35.8 \\
36.4\end{array}$ & \\
\hline 17 & $\begin{array}{r}8 \\
10 \\
13 \\
22 \\
37\end{array}$ & 1500 & $\begin{array}{l}1420 \\
1400 \\
1500 \\
2010\end{array}$ & $\begin{array}{l}42 \\
42 \\
44\end{array}$ & $\begin{array}{l}113.9 \\
114.7 \\
112.8 \\
114.2\end{array}$ & $\begin{array}{l}51.0 \\
62.0 \\
\\
55.4 \\
83.6\end{array}$ & $\begin{array}{l}62.9 \\
52.7 \\
57.4 \\
30.6\end{array}$ & $\begin{array}{l}42.9 \\
38.2 \\
\\
34.4 \\
31.7\end{array}$ & $\begin{array}{l}29.4 \\
30.2 \\
32.1 \\
30.8\end{array}$ & $\begin{array}{r}13.5 \\
8.0 \\
\\
2.3 \\
0.9\end{array}$ & $\begin{array}{r}10.1 \\
28.3 \\
9.8 \\
-5.4 \\
13.9\end{array}$ & $\begin{array}{l}7.455 \\
7.362 \\
7.386 \\
7.405\end{array}$ & $\begin{array}{l}40 \\
18\end{array}$ & $\begin{array}{l}36.4 \\
37.1 \\
36.3 \\
36.8\end{array}$ & $\begin{array}{l}\text { post-respiratory } \\
\text { distress syndrome }\end{array}$ \\
\hline 18 & $\begin{array}{r}1 \\
2 \\
9 \\
20 \\
28\end{array}$ & 1520 & $\begin{array}{l}1520 \\
1500 \\
1880 \\
2130\end{array}$ & $\begin{array}{l}40 \\
44 \\
45\end{array}$ & $\begin{array}{r}96.8 \\
102.2 \\
110.1\end{array}$ & $\begin{array}{r}92.7 \\
82.0 \\
105.0\end{array}$ & $\begin{array}{r}4.1 \\
20.2 \\
5.1\end{array}$ & $\begin{array}{l}39.1 \\
44.0 \\
43.6\end{array}$ & $\begin{array}{l}45.0 \\
39.8 \\
33.5\end{array}$ & $\begin{array}{r}-5.9 \\
4.2 \\
10.1\end{array}$ & $\begin{array}{r}1.4 \\
12.3 \\
12.8 \\
-0.8 \\
-7.4\end{array}$ & $\begin{array}{l}7.348 \\
7.470 \\
7.367\end{array}$ & $\begin{array}{l}26 \\
26 \\
28\end{array}$ & $\begin{array}{l}36.8 \\
36.6 \\
36.8\end{array}$ & \\
\hline 19 & $\begin{array}{l}1 \\
5\end{array}$ & 1600 & $\begin{array}{l}1600 \\
1460\end{array}$ & $\begin{array}{l}43 \\
43\end{array}$ & $\begin{array}{l}115.0 \\
112.8\end{array}$ & $\begin{array}{l}55.0 \\
53.0\end{array}$ & $\begin{array}{l}60.0 \\
59.8\end{array}$ & $\begin{array}{l}36.0 \\
44.6\end{array}$ & $\begin{array}{l}26.1 \\
30.4\end{array}$ & $\begin{array}{r}9.9 \\
14.2\end{array}$ & $\begin{array}{l}-3.0 \\
13.1\end{array}$ & $\begin{array}{l}7.410 \\
7.363\end{array}$ & $\begin{array}{l}45 \\
42\end{array}$ & $\begin{array}{l}36.5 \\
36.5\end{array}$ & $\begin{array}{l}2 \text { Weight at time } \\
\text { of study }\end{array}$ \\
\hline
\end{tabular}




\begin{tabular}{|c|c|c|c|c|c|c|c|c|c|c|c|c|c|c|c|}
\hline & $\begin{array}{l}13 \\
21\end{array}$ & & $\begin{array}{l}1610 \\
1920\end{array}$ & 45 & $\begin{array}{l}106.9 \\
117.9\end{array}$ & $\begin{array}{l}56.2 \\
57.6\end{array}$ & $\begin{array}{l}50.7 \\
60.0\end{array}$ & $\begin{array}{l}47.0 \\
39.4\end{array}$ & $\begin{array}{l}36.1 \\
35.3\end{array}$ & $\begin{array}{r}10.9 \\
4.1\end{array}$ & 16.6 & 7.368 & $\begin{array}{l}33 \\
37\end{array}$ & $\begin{array}{l}37.0 \\
36.6\end{array}$ & \\
\hline 20 & $\begin{array}{r}6 \\
12 \\
15\end{array}$ & 1640 & $\begin{array}{l}1620 \\
1660 \\
1820\end{array}$ & $\begin{array}{l}41 \\
41\end{array}$ & $\begin{array}{r}103.0 \\
97.5 \\
98.2\end{array}$ & $\begin{array}{l}68.7 \\
59.1 \\
76.9\end{array}$ & $\begin{array}{l}34.3 \\
38.4 \\
21.3\end{array}$ & $\begin{array}{l}44.3 \\
43.6 \\
44.5\end{array}$ & $\begin{array}{l}40.8 \\
40.0 \\
44.0\end{array}$ & $\begin{array}{l}3.5 \\
3.6 \\
0.5\end{array}$ & $\begin{array}{r}-10.5 \\
2.5 \\
7.4 \\
\end{array}$ & $\begin{array}{l}7.495 \\
7.500 \\
7.415\end{array}$ & $\begin{array}{l}35 \\
38\end{array}$ & $\begin{array}{l}37.4 \\
36.8 \\
36.7\end{array}$ & $\begin{array}{l}\text { post-respiratory } \\
\text { distress syndrome }\end{array}$ \\
\hline 21 & $\begin{array}{l}2 \\
4\end{array}$ & 1758 & 1670 & 42 & 100.8 & 79.7 & 21.6 & 40.4 & 40.0 & 0.4 & $\begin{array}{l}-7.2 \\
-3.1\end{array}$ & 7.35 & 47 & 36.5 & \\
\hline 22 & $\begin{array}{r}7 \\
8 \\
14 \\
20 \\
22\end{array}$ & 1760 & $\begin{array}{l}1650 \\
1680 \\
1860 \\
1860\end{array}$ & $\begin{array}{l}42 \\
43 \\
43 \\
43\end{array}$ & $\begin{array}{l}104.5 \\
113.2 \\
114.1 \\
116.8\end{array}$ & $\begin{array}{l}48.7 \\
54.7 \\
73.9 \\
66.0\end{array}$ & $\begin{array}{l}55.8 \\
58.5 \\
40.2 \\
50.8\end{array}$ & $\begin{array}{l}46.0 \\
46.6 \\
36.2 \\
43.1\end{array}$ & $\begin{array}{l}36.2 \\
30.3 \\
29.3 \\
29.4\end{array}$ & $\begin{array}{r}9.8 \\
16.3 \\
6.9 \\
13.7\end{array}$ & $\begin{array}{l}-5.1 \\
-4.6 \\
-3.0 \\
-1.9 \\
-6.0\end{array}$ & $\begin{array}{l}7.302 \\
7.375 \\
7.395 \\
7.320\end{array}$ & $\begin{array}{l}30 \\
21 \\
19 \\
19\end{array}$ & $\begin{array}{l}36.7 \\
36.1 \\
36.5 \\
36.5\end{array}$ & $\begin{array}{l}\text { post-respiratory } \\
\text { distress syndrome }\end{array}$ \\
\hline 23 & $\begin{array}{r}2 \\
3 \\
9 \\
18 \\
24\end{array}$ & 1770 & $\begin{array}{l}1800 \\
1800 \\
1640 \\
1800 \\
2110\end{array}$ & $\begin{array}{l}43 \\
44 \\
45\end{array}$ & $\begin{array}{l}107.6 \\
109.3 \\
116.0 \\
104.8 \\
108.0\end{array}$ & $\begin{array}{l}92.0 \\
74.7 \\
70.8 \\
68.6 \\
96.5\end{array}$ & $\begin{array}{l}15.6 \\
34.6 \\
45.2 \\
36.2 \\
11.5\end{array}$ & $\begin{array}{l}39.0 \\
35.9 \\
48.2 \\
44.6 \\
40.2\end{array}$ & $\begin{array}{l}35.4 \\
32.4 \\
27.9 \\
37.3 \\
34.2\end{array}$ & $\begin{array}{r}3.6 \\
3.5 \\
20.3 \\
7.3 \\
6.0\end{array}$ & $\begin{array}{r}10.2 \\
-5.0 \\
9.5 \\
5.8 \\
3.7\end{array}$ & $\begin{array}{l}7.475 \\
7.420 \\
7.453 \\
7.443\end{array}$ & $\begin{array}{l}26 \\
29 \\
26 \\
38 \\
22\end{array}$ & $\begin{array}{l}36.4 \\
36.2 \\
36.2 \\
36.8 \\
37.1\end{array}$ & \\
\hline 24 & $\begin{array}{l}1 \\
2 \\
3 \\
5 \\
8\end{array}$ & 2610 & $\begin{array}{l}2610 \\
2500 \\
2420 \\
2350 \\
2360\end{array}$ & 46 & $\begin{array}{l}121.0 \\
109.2\end{array}$ & $\begin{array}{l}25.4 \\
30.8 \\
43.1 \\
65.2 \\
85.0\end{array}$ & $\begin{array}{l}55.8 \\
24.2\end{array}$ & $\begin{array}{l}55.5 \\
50.0 \\
38.8 \\
44.0 \\
37.6\end{array}$ & $\begin{array}{l}31.6 \\
33.4 \\
33.5\end{array}$ & $\begin{array}{r}22.9 \\
\\
10.6 \\
4.1\end{array}$ & $\begin{array}{l}11.5 \\
-5.6\end{array}$ & $\begin{array}{l}7.267 \\
7.392 \\
7.445\end{array}$ & $\begin{array}{l}33 \\
28 \\
37 \\
\\
37\end{array}$ & $\begin{array}{l}36.7 \\
38.3 \\
36.9 \\
36.8 \\
37.3\end{array}$ & $\begin{array}{l}\text { respiratory distress } \\
\text { syndrome }\end{array}$ \\
\hline
\end{tabular}

$\mathrm{PA}_{\mathrm{O}_{2}}: \quad$ Alveolar oxygen tension.

$\mathrm{PaO}_{2}: \quad$ Arterial oxygen tension.

$\mathrm{AaD}_{\mathrm{O}_{2}}$ : Alveolar-arterial oxygen gradient.

$\mathrm{PaCO}_{2}$ : Arterial carbon dioxide tension.

$\mathrm{PA}_{\mathrm{CO}_{2}}$ : Alveolar carbon dioxide tension.

aAD $\mathrm{AO}_{2}$ : Arterial-alveolar carbon dioxide gradient.

$\mathrm{uAD}_{\mathrm{N}_{2}}: \quad$ Urinary-alveolar nitrogen gradient.

Hct: Hematocrit.

f: $\quad$ Breaths/min

$\dot{\mathrm{V}}_{\mathrm{CO}_{2}}: \quad \mathrm{CO}_{2}$ production, $\mathrm{ml} / \mathrm{min}$ STPD.

$\dot{\mathrm{V}}_{\mathrm{O}_{2}}: \quad \mathrm{O}_{2}$ consumption, $\mathrm{ml} / \mathrm{min}$ STPD.
R: Respiratory exchange ratio

$\mathrm{V}_{\mathrm{DP}}$ : Physiologic dead space.

$\mathrm{V}_{\mathrm{T}}$ : Tidal volume.

$\mathrm{V}_{\mathrm{D}} / \mathrm{V}_{\mathrm{T}}$ : Physiologic dead space/tidal volume ratio.

$\dot{V}_{E}$ : $\quad$ Minute ventilation.

$\dot{\mathrm{V}}_{\mathrm{A}}$ : $\quad$ Alveolar ventilation, $\mathrm{ml} / \mathrm{min}$ BTPS

$\dot{V}_{\text {DP }}$ : Physiologic dead space ventilation.

Anatomic dead space.

$\mathrm{V}_{\mathrm{D}_{\text {anat. }}:}$ Anatomic dead space.

$V_{D_{a l y}}: \quad$ Alveolar dead space $\left(V_{D p}-V_{\text {Danat }}\right)$

$\hat{\mathrm{V}}_{\mathrm{A}} / \dot{\mathrm{V}}_{\mathrm{O}_{2}}$ : Alveolar ventialtion/oxygen consumption. 
Table II. Serial studies on premature infants of dead space and related parameters ${ }^{1}$

\begin{tabular}{|c|c|c|c|c|c|c|c|c|c|c|c|c|c|c|c|c|c|}
\hline \multirow{2}{*}{$\begin{array}{l}\text { Case } \\
\text { No. }\end{array}$} & \multirow{2}{*}{$\begin{array}{l}\text { Age }^{2} \\
\text { days }\end{array}$} & \multirow{2}{*}{$\begin{array}{c}f \\
\min \end{array}$} & \multirow{2}{*}{\multicolumn{2}{|c|}{$\frac{\dot{\mathrm{V}}_{\mathrm{CO}_{2}}-\dot{\mathrm{V}}_{\mathrm{O}_{2}}}{\mathrm{ml} / \mathrm{min}}$}} & \multirow[t]{2}{*}{$\mathrm{R}$} & \multirow[t]{2}{*}{$\mathrm{V}_{\mathrm{DP}}$} & \multirow{2}{*}{$\frac{V_{T}}{1}$} & \multirow[t]{2}{*}{$\mathrm{V}_{\mathrm{D}} / \mathrm{V}_{\mathrm{T}}$} & \multirow[t]{2}{*}{$\dot{\mathrm{V}}_{\mathrm{E}}$} & \multirow{2}{*}{\multicolumn{2}{|c|}{$\frac{\dot{\mathrm{V}}_{\mathrm{A}}}{\mathrm{ml} / \mathrm{r}}$}} & \multirow[t]{2}{*}{$\mathrm{V}_{\mathrm{DP}}$} & \multirow{2}{*}{$\frac{\mathrm{V}_{D_{\text {anat. }}}}{\mathrm{ml}}$} & \multirow[t]{2}{*}{$\mathrm{V}_{D_{\text {alv. }}}$} & \multirow[t]{2}{*}{$\dot{\mathrm{V}}_{\mathrm{A}} / \dot{\mathrm{V}}_{\mathrm{O}_{2}}$} & \multicolumn{2}{|c|}{$\%$ alveoli } \\
\hline & & & & & & & & & & & & & & & & $\begin{array}{l}\text { Not } \\
\text { per- } \\
\text { fused }\end{array}$ & $\begin{array}{c}\text { Not } \\
\text { venti- } \\
\text { lated }\end{array}$ \\
\hline \multirow[t]{2}{*}{2} & 6 & 80 & 4.2 & 7.2 & 0.58 & 1.9 & 3.1 & 0.61 & 248 & 114 & 96 & 150 & 1.2 & 0.7 & 15.8 & 50 & 30 \\
\hline & 40 & 65 & 9.9 & 12.8 & 0.81 & 4.7 & 7.8 & 0.61 & 507 & 244 & 286 & 306 & 4.4 & 0.3 & 19.1 & 5 & 15 \\
\hline \multirow[t]{2}{*}{4} & 10 & 41 & 6.3 & 7.2 & 0.88 & 4.3 & 6.9 & 0.62 & 283 & 135 & 164 & 172 & 4.0 & 0.3 & 18.8 & 5 & 40 \\
\hline & 44 & 31 & 9.5 & 10.7 & 0.89 & 5.4 & 12.1 & 0.45 & 376 & 240 & 136 & 168 & 4.4 & 1.0 & 22.1 & 5 & 10 \\
\hline \multirow[t]{3}{*}{6} & 2 & 55 & 4.1 & 8.0 & 0.51 & 8.2 & 9.5 & 0.86 & 522 & 91 & & 450 & & & 11.4 & & \\
\hline & 24 & 47 & 9.4 & 9.6 & 0.98 & 7.6 & 11.3 & 0.68 & 530 & 205 & & 358 & & & 21.4 & & \\
\hline & 30 & 65 & 13.3 & 17.5 & 0.76 & 5.0 & 10.4 & 0.48 & 676 & 344 & 318 & 325 & 4.9 & 0.1 & 19.7 & 0 & 15 \\
\hline \multirow[t]{3}{*}{8} & 6 & 49 & 6.1 & 5.9 & 1.05 & 5.1 & 7.1 & 0.71 & 348 & 120 & 219 & 250 & 4.5 & 0.6 & 20.4 & 20 & 25 \\
\hline & 26 & 44 & 9.4 & 11.2 & 0.84 & 8.5 & 12.4 & 0.69 & 548 & 208 & 361 & 374 & 8.2 & 0.3 & 18.6 & 0 & 30 \\
\hline & 40 & 44 & 12.4 & 14.2 & 0.87 & 8.4 & 13.3 & 0.63 & 585 & 263 & 370 & 370 & 8.4 & 0.0 & 18.5 & 0 & 15 \\
\hline \multirow[t]{3}{*}{10} & 2 & 52 & 4.5 & 7.9 & 0.57 & 4.4 & 5.9 & 0.75 & 306 & 93 & 208 & 230 & 4.0 & 0.4 & 11.7 & 20 & 15 \\
\hline & 5 & 44 & 5.7 & 8.1 & 0.70 & 5.4 & 8.2 & 0.66 & 361 & 152 & 236 & 236 & 5.4 & 0.0 & 18.8 & 0 & 10 \\
\hline & 26 & 47 & 10.0 & 12.6 & 0.79 & 5.0 & 13.1 & 0.38 & 615 & 246 & 197 & 235 & 4.2 & 0.8 & 19.5 & 5 & 15 \\
\hline \multirow[t]{3}{*}{12} & 1 & 55 & 6.9 & 10.7 & 0.65 & 7.0 & 8.8 & 0.80 & 485 & 150 & 380 & 387 & 6.9 & 0.1 & 14.0 & 0 & $>50$ \\
\hline & 10 & 57 & 6.5 & 7.3 & 0.89 & 5.5 & 9.8 & 0.73 & 428 & 138 & 296 & 313 & 5.2 & 0.3 & 18.9 & 5 & 15 \\
\hline & 24 & 44 & 8.9 & 10.6 & 0.84 & 7.0 & 12.1 & 0.58 & 410 & 202 & 295 & 308 & 6.7 & 0.3 & 19.0 & 5 & 5 \\
\hline \multirow[t]{2}{*}{15} & 13 & 59 & 7.9 & 8.9 & 0.89 & 9.5 & 11.6 & 0.82 & 684 & 153 & 523 & 560 & 8.9 & 0.6 & 17.2 & 10 & 7 \\
\hline & 19 & 45 & 10.6 & 16.6 & 0.64 & 6.2 & 11.2 & 0.55 & 502 & 267 & 274 & 277 & 6.1 & 0.05 & 16.1 & 0 & 5 \\
\hline \multirow[t]{2}{*}{20} & 6 & 35 & 7.1 & 9.6 & 0.74 & 9.1 & 12.4 & 0.73 & 434 & 139 & 307 & 317 & 8.8 & 0.3 & 14.5 & 5 & 15 \\
\hline & 15 & 30 & 11.1 & 15.9 & 0.70 & 2.8 & 2.8 & 0.32 & 265 & 215 & 84 & 84 & 2.8 & 0.0 & 13.5 & 0 & 10 \\
\hline \multirow[t]{2}{*}{22} & 8 & 68 & 8.7 & 13.0 & 0.67 & 6.5 & 8.5 & 0.77 & 580 & 163 & 374 & 442 & 5.5 & 1.0 & 12.5 & 20 & 50 \\
\hline & 22 & 73 & 15.0 & 17.0 & 0.88 & 11.6 & 15.2 & 0.76 & 1110 & 298 & 725 & 850 & 9.9 & 1.7 & 17.4 & 30 & 25 \\
\hline \multirow[t]{5}{*}{24} & 1 & 72 & 11.2 & 22.9 & 0.49 & 10.5 & 12.5 & 0.84 & 906 & 179 & 550 & 706 & 7.6 & 2.7 & 16.0 & $>25$ & $>50$ \\
\hline & 2 & 81 & 12.7 & 22.7 & 0.56 & 10.3 & 12.5 & 0.82 & 1012 & 219 & & 830 & & & 17.2 & & \\
\hline & 3 & 45 & 14.4 & 23.7 & 0.61 & 6.4 & & & & & & & & & & & \\
\hline & 5 & 46 & 12.1 & 19.3 & 0.63 & 9.8 & 14.5 & 0.68 & 620 & 236 & 268 & 455 & 5.8 & 3.0 & 19.5 & 25 & 30 \\
\hline & 8 & 35 & 14.5 & 22.7 & 0.64 & 6.0 & 13.9 & 0.43 & 486 & 332 & 128 & 210 & 5.1 & 0.9 & 22.9 & 10 & 10 \\
\hline
\end{tabular}

${ }^{1}$ See footnote 1 in table I for symbols.

${ }^{2}$ At time of studies. 


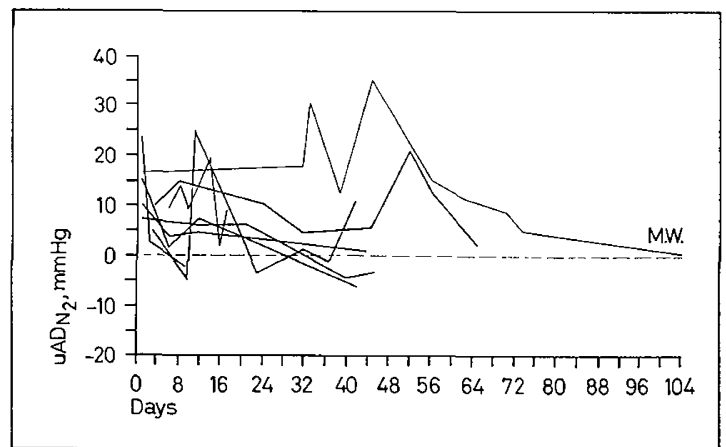

Fig. 1. Serial determination of $\mathrm{uAD}_{\mathrm{N}_{2}}$ in infants with birth weights under $1200 \mathrm{~g}$. M.W. was an infant with Mikity-Wilson disease.

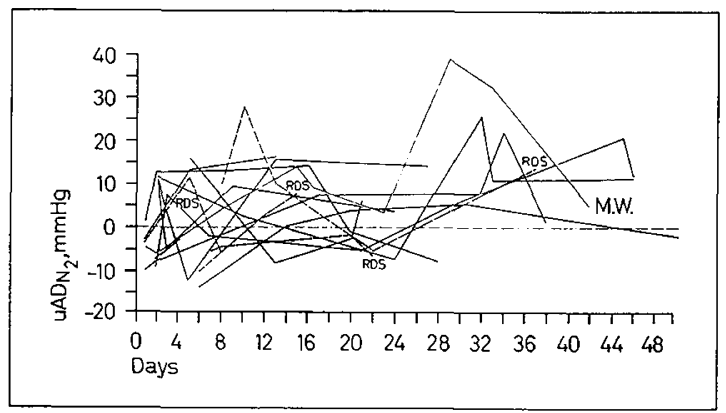

Fig.2. Serial studies of $\mathrm{uAD}_{\mathrm{N}_{2}}$ in infants with birth weights over $1200 \mathrm{~g}$. RDS infants had hyaline membranes disease. M.W. had Mikity-Wilson disease.

alveoli, with a similar number nonventilated, utilizing the method of Severinghaus and Stupfel [6]. It should be emphasized that the sequence of relations is the result of several factors: increasing physical size of the lungs, changing $\vec{V}_{\mathrm{A}} / \dot{Q}$ and metabolic rates, and respiratory rates.

\section{Discussion}

The data confirm the conclusions of previous studies [1] from this laboratory in which high values for $\mathrm{AaD}_{\mathrm{O}_{2}}$ and $\mathrm{aAD}_{\mathrm{CO}_{2}}$ were exhibited in the smaller premature infants. The higher values for $\mathrm{aDD}_{\mathrm{CO}_{2}}$ reflected areas of increased ventilation-perfusion ratio, and the $\mathrm{AaD}_{2}$ valucs during air breathing indicated abnormalities in oxygenation as a result of shunting, diffusion, or alveoli with a low ventilation-perfusion ratio. An increased $u A D_{N_{2}}$ develops in alveoli with a low $\dot{V}_{\mathrm{A}} / \dot{Q}$, and this test may thus be used to separate some of the compo-

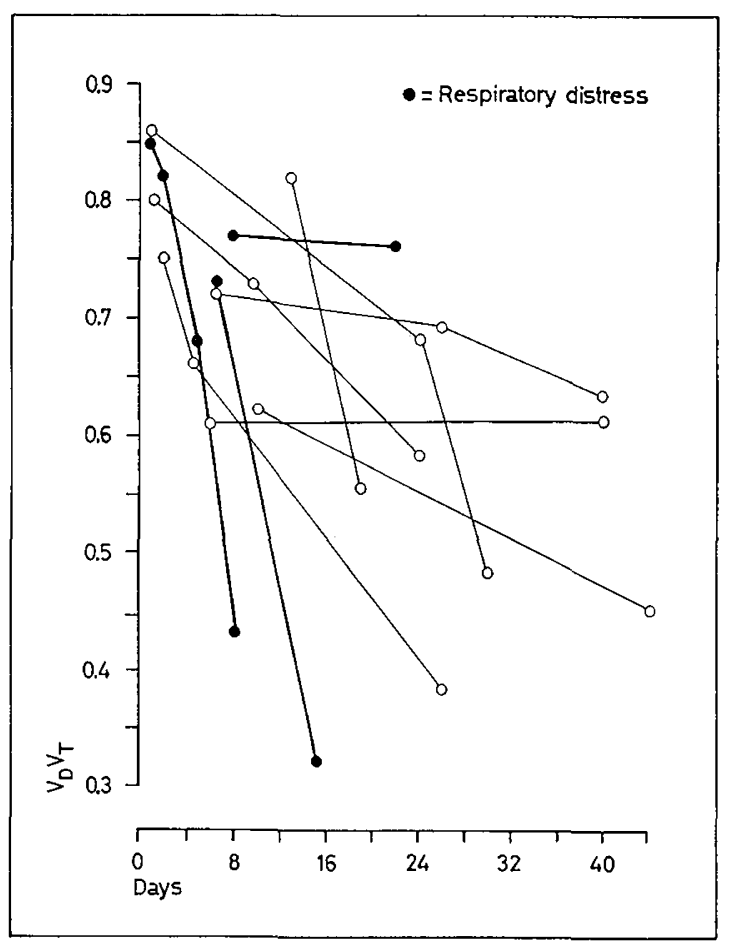

Fig.3. Serial studies of $\mathrm{V}_{\mathrm{D}} / \mathrm{V}_{\mathrm{T}}$ in infants with and without hyaline membrane disease.

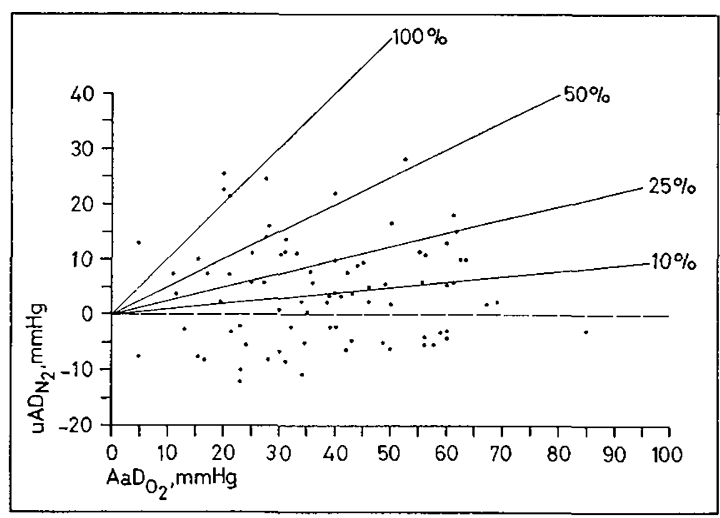

Fig.4. Urinary-alveolar nitrogen gradient plotted with respect to alveolar-arterial oxygen gradient. Guidelines indicate $u \mathrm{AD}_{\mathrm{N}_{2}}$ as a percent of simultaneously determined $\mathrm{AaD}_{\mathrm{O}_{2}}$.

nents of $\mathrm{AaD}_{\mathrm{O}_{2}}$. Large daily variations in $\mathrm{uAD}_{\mathrm{N}_{2}}$ were noted with a tendency toward lessening as the infant aged. The present data, together with the studies of Thibeault et al. [8], in which considerable prolonged 
intrapulmonary shunting was demonstrated, indicate that there are at least two reasons for the high $\mathrm{AaD}_{\mathrm{O} 2}$ in the premature infant: shunting and $\dot{V}_{\Lambda} / \dot{Q}$ unevenness. Analysis of the data shown in figure 4 indicates that a major component of the oxygen gradient is not due to $\dot{V}_{A} / \dot{Q}$ unevenness. The results are consistent with the concept that the infant has atelectasis or nonventilated alveoli that gradually return to normal. These conclusions are in contradistinction to the opinion of LEDBETTER et al. [3] that in the full-term infant, the major component of $\mathrm{AaD}_{(\mathrm{O}) \text { is }} \dot{\mathrm{V}}_{\mathrm{A}} / \dot{\mathrm{Q}}$ unevenness, although the studies of NELSON et al. [5] favor shunting to be the major abnormality. The possibility that part of the shunting is intracardiac (foramen ovale) cannot be excluded.

The expansion of the premature infant's lung from its initial fluid-filled state does not proceed in a smooth, orderly fashion. A pattern of extrauterine adaptation of the lungs, based on physiologic observation, is emerging. Initially, these infants have a large thoracic gas volume that decreases in the first week of life [9] and is associated with gas trapping [7], shunting [8], and ventilation-perfusion unevenness. As the lungs increase in size with the growth of the infant, atelectasis and shunting persist and pulmonary development progresses with evidence of areas of both high and low values for $\dot{\mathrm{V}}_{\mathrm{A}} / \dot{\mathrm{Q}}$. Thus, the tendency of these small premature infants to develop respiratory difficulty from relatively minor stresses such as distended stomach and feeding is readily explicable on the basis of altered pulmonary physiology. Data on infants with hyaline membrane disease suggest, qualitatively at least, that they have many of the same defects.

\section{Summary}

The lung of the premature infant is not a physiologically homogenous organ with respect to ventilation and perfusion. Areas of high and low $\dot{V}_{\mathrm{A}} / \dot{Q}$ contributing to aAD $\mathrm{CO}_{2}$ and $\mathrm{uAD} \mathrm{N}_{2}$, and a telectasis reflected in $\mathrm{AaD}_{\mathrm{O}_{2}}$, exist simultaneously. Overperfusion of poorly ventilated alveoli occurs as atelectasis resolves, but does not appear to be a major reason for the persistent $\mathrm{AaD}_{\mathrm{O}_{2}}$.
References and Notes

1. Farhi, L. E.; Edwards, A.W.T. and Homma, T.: Determination of dissolved $\mathrm{N}_{2}$ in blood by gas chromatography and (a-A) $\mathrm{N}_{2}$ difference. J.appl. Physiol. 18: 97 (1963).

2. Krauss, A.N. and Auld, P.A.M.: Unpublished data.

3. Ledbetter, M.K.; Homma, T. and Farhi, L.E.: Readjustment in distribution of alveolar ventilation and lung perfusion in the newborn. Pediatrics 40: 940 (1967).

4. Mead, J.: Control of respiratory frequency. J. appl. Physiol. 15: 325 (1960).

5. Nelson, N. M.; Prod'hom, L.S.; Cherry, R.B.; Lipsitz, P.J. and SмiтH, C. A.: Pulmonary function in the newborn infant; the alveolar arterial oxygen gradient. J.appl. Physiol. 18: 534 (1963).

6. Severinghaus, J.W. and Stupfel, M.: Alveolar dead space as an index of distribution of blood flow in pulmonary capillaries. J.appl. Physiol. 10: 335 (1957).

7. Thibeault, D.W.; Poblete, E. and Auld, P.A.M.: Alveolar-arterial $\mathrm{O}_{2}$ and $\mathrm{CO}_{2}$ differences and their relation to lung volume in the newborn. Pediatrics 41: 574 (1968).

8. Thibeault, D.W.; Poblete, E. and Auld, P.A.M.: Alveolar-arterial oxygen difference in premature infants breathing 100 per cent oxygen. J.Pediat. 71: 814 (1967).

9. Thibeault, D.W.; Wong, M.M. and Auld, P.A.M.: Thoracic gas volume changes in premature infants. Pediatrics 40: 403 (1967).

10. Yellow Springs Instrument Company, Yellow Springs, Ohio.

11. Supported by US Public Health Research Grant HD-02644-01 and the Educational Foundation of America. Dr. Auld holds an Investigatorship of the Health Research Council of New York (under Contract 1-236).

12. Requests for reprints should beaddressed to P.A.M. Auld, M.D., Department of Pediatrics, The New York Hospital-Cornell Medical Center, 525 East 68th Street, New York, N.Y. 10021 (USA). 